\title{
Correlation between Nasoalveolar Molding and Surgical, Aesthetic, Functional and Socioeconomic Outcomes Following Primary Repair Surgery: a Systematic Review
}

\author{
Sophie Maillard ${ }^{1}$, Jean-Marc Retrouvey ${ }^{1}$, Mairaj K. Ahmed ${ }^{2}$, Peter J. Taub ${ }^{3}$ \\ ${ }^{1}$ Division of Orthodontics, Faculty of Dentistry, McGill University, Montreal, Quebec, Canada. \\ ${ }^{2}$ Departments of Dentistry/Oral/Maxillofacial Surgery, Otolaryngology, and Surgery. Mount Sinai Cleft and Craniofacial \\ Center, Icahn School of Medicine at Mount Sinai, New York, USA. \\ ${ }^{3}$ Departments of Dentistry, Pediatrics, Surgery and Medical Education, Mount Sinai Cleft and Craniofacial Center, Icahn \\ School of Medicine at Mount Sinai, New York, USA.
}

\author{
Corresponding Author: \\ Sophie Maillard \\ Division of Orthodontics, Faculty of Dentistry \\ McGill University \\ Strathcona Bldg. Room M-72, 3640 University Street, QC H3A 0C7, Montreal, Quebec \\ Canada \\ Phone: 1-514-398-7203 Ext. 094894 (Lab) \\ Fax: 1-514-398-8900 \\ E-mail: sophie.maillard@mail.com
}

\begin{abstract}
Objectives: The authors performed a systematic review to evaluate the potential beneficial effects of the nasoalveolar molding appliance on nonsyndromic unilateral clefts of the lip and/or palate prior to primary lip repair.

Material and Methods: A literature search was performed using three electronic databases (PubMed, Embase, Web of Science) and three journals ("Cleft Palate-Craniofacial Journal", "Plastic and Reconstructive Surgery Journal" and "American Journal of Orthodontics and Dentofacial Orthopaedic") from January 1980 to April 2017. Data extraction was performed with tables treating different subjects: surgical, aesthetical, functional, socio-economical effects of nasoalveolar molding (NAM) appliances and the evolution of NAM appliances, especially three-dimensional technology.

Results: Of the 145 articles retrieved in the literature surveys, 28 were qualified for the final analysis and 20 studies were excluded because of their small sample size (less than 10 patients) and/or too long follow-up (exceeded 18 months). Four randomized controlled trials were available. Although literature allowed discussing the short-term benefits of NAM appliance and the three-dimensional technology, scientific evidence is lacking.

Conclusions: Based on the results, nasoalveolar molding appliances have positive surgical, aesthetical, functional and socioeconomical effects on unilateral clefts of the lip and/or palate treatment before the primary repair surgeries. Three-dimensional technology results in a more efficient and predictable nasoalveolar molding appliance treatment. However, nasoalveolar molding appliance effect in a short term remains unclear with the available literature. Further studies that integrate threedimensional technology in a large scale are still needed.
\end{abstract}

Keywords: cleft lip; cleft palate; orthodontic appliances; newborn infant.

Accepted for publication: 29 May 2017

To cite this article:

Maillard S, Retrouvey JM, Ahmed MK, Taub PJ.

Correlation between Nasoalveolar Molding and Surgical, Aesthetic, Functional and Socioeconomic Outcomes Following Primary Repair Surgery: a Systematic Review

J Oral Maxillofac Res 2017;8(3):e2

URL: http://www.ejomr.org/JOMR/archives/2017/3/e2/v8n3e2.pdf

doi: $10.5037 /$ jomr.2017.8302 


\section{INTRODUCTION}

Clefts of the lip and palate result from a fusion failure of the left and right maxillary prominences during the $6^{\text {th }}$ and $12^{\text {th }}$ weeks of gestation. The resultant congenital deformity leads to malformation of the upper lip, nose and alveolar and results in functional disabilities (i.e. mastication, feeding, speaking). This affects approximately 1/700 live birth in North America and the majority of clefts are unilateral and non-syndromic [1]

Two general strategies are used to close the lip and correct the nasal asymmetry. One approach involves lip and nose repair around three months of age irrespective of the size of the alveolar gap and may be followed by secondary correction of any residual deformity sometime during childhood. The second approach utilizes presurgical orthopaedic molding early after birth for approximately three months and must be performed prior to primary repair surgeries. With the latter, orthodontists and surgeons aim to provide symmetry and elongation to the deformed nasal cartilage and reduce the severity of the cleft palate prior to the initial surgical intervention [2].

Since the 1950s, McNeil [프, 4$]$ described the first intraoral presurgical orthopaedic appliance able to stimulate the tissue growth and reduce the width of the alveolar and palatal cleft. Then, clinicians have proposed several presurgical appliances to mold the alveolar arch and reduce lip and palate cleft. First, passive appliances, such as the Hotz plate, composed of a simple plate, aim to create alveolar alignment by spontaneous development of the segments without external force. Then, in the 1980s, active orthopaedics, such as the Latham appliance, are retained by surgically installed pins and delivers controlled forces to reduce the cleft gap and align the alveolar arch. Finally, in the 1990, Grayson used Matsuo et al. [5] concept and described a third category of presurgical infant appliances, semi-active, called the nasoalveolar molding (NAM) appliance. The appliance is composed of a passive alveolar plate with one (for unilateral cleft lip and/or palate [UCLP]) or two (for bilateral cleft lip and/or palate [BCLP]) nasal stents. NAM is the first appliance able to mold presurgically the alveolar arch into a predictable preferred alignment and correct the nasal cartilage at the same time [ $[\underline{6}, 7]$. To achieve its goal, the appliance is introduced within the first two weeks of life in order to benefit from the maximum growth potential. It must be worn full-time until the alveolar cleft is sufficiently narrowed and the lip segments are brought together. A nasal stent extending from the intraoral plate may be added to improve nasal symmetry. Primary lip repair is then performed once the effect of NAM is deemed adequate, usually after the 18 months. This surgery is more or less invasive depending on the efficiency of the NAM.

Recent reviews have been conducted to evaluate the efficacy of the presurgical orthopaedic treatment on patients with nonsyndromic unilateral clefting of the lip and/or palate. However the heterogeneity of these studies [8-12] prevents the construction of substantial evidence of the NAM effect (Table 1). Moreover, many studies evaluate the potential beneficial effects of the NAM appliance after the primary lip repair, whereas the efficiency of the appliance itself should be assessed prior to the primary lip repair (during the first 18 months).

The aim of this study was to undertaken a systematic review to compare the surgical, aesthetic, functional and socio-economical effects before the primary lip repair between patients with nonsyndromic unilateral clefting of the lip and/or palate treated either with the NAM appliance or with other presurgical appliances

Table 1. Heterogeneity between studies

\begin{tabular}{l|l}
\hline \multicolumn{1}{c}{ Variables } & \multicolumn{1}{c}{ Heterogeneity of studies } \\
\hline Different study designs & Retrospective [36,44], prospective [32,34], randomised [58,64]. \\
\hline Different overall aims & Assessment of the molding of the nasal cartilage [27,44], molding of the alveolar possesses [40,42]. \\
\hline Mixed criteria for evaluating results & $\begin{array}{l}\text { Landmarks on casts [26], cephalometry, facial photographs [43,44], photographs on cast [45,46], } \\
\text { three-dimensional optical scanner on the cast [64], three-dimensional optical facial scanner [62,63]. }\end{array}$ \\
\hline Inadequate sample sizes & Small sample size [17,19], without control group [27]. \\
\hline Inconsistent follow-up period & Before the primary surgery (during the first 18 months), after the primary surgeries [22,23,26]. \\
\hline Heterogeneity of appliances & Hotz plate [41,43], Grayson NAM [40]. \\
\hline Variable age of the patient & When the appliance was used, when primary surgeries were performed. \\
\hline Mixed level of surgeon experience & \\
{$[12]$} & \\
\hline
\end{tabular}

$\mathrm{NAM}=$ nasoalveolar molding. 
or without any presurgical treatment. The objective of the study was also to highlight complications and inefficiency of the NAM appliance. The final aim of the systematic review was to introduce the evolution of the NAM appliance technique, especially ones involving three-dimensional technology that have the potential to overcome some of the inefficiencies of the current NAM appliance approach will be introduced.

\section{MATERIAL AND METHODS}

The materials and methods of the literature employed by the authors in the systematic reviews are based on comprehensive search strategies that have been discussed and standardized.

\section{Protocol}

PRISMA-P (2015) recommendations were used to methodically build this review [13].

\section{Focus questions}

The four PICO elements (population, intervention, comparison and outcome) were employed to construct the systematic reviews. The focus questions:

1. What are the outcomes of NAM prior to primary repair surgeries on the UCLP compare to the outcomes without NAM or with others appliances?

2. What are the applications of three-dimensional technology in NAM approach nowadays?

\section{Search strategy}

An electronic search of three databases (PubMed, Medline, Web of Science) was performed and included articles from January 1980 to April 2017. The research terms used to identify articles discussing NAM appliance were: MeSH terms "cleft palate" OR "cleft lip" AND non-MeSH terms: "nasoalveolar molding, "presurgical nasoalveolar molding", "presurgical orthopaedics", "presurgical orthopaedic appliance". Beside manual search in 3 journals was conducted: "Cleft Palate-Craniofacial Journal", "Plastic and Reconstructive Surgery Journal" and "American Journal of Orthodontics and Dentofacial Orthopaedic".

\section{Type of study}

The review includes all human prospective and retrospective follow-up, cohort studies, case series and randomized control studies related to NAM appliance outcomes on the UCLP.

\section{Domain being studies}

Surgical, aesthetic, functional, socio-economical effects of NAM appliances and evolution of NAM appliances, especially $\mathrm{CAD} / \mathrm{CAM}$ technologies were the domain being studied in this systematic review. The outcomes were compared to results on patients treated with other presurgical appliances or patients who did not receive any presurgical treatment before the primary repair surgeries.

\section{Inclusion criteria}

Search criteria include a minimum of ten patients and a follow-up not exceeding 18 months, before the first primary repair, corresponding on the period when the facial growth is the most important. Within these studies, only the ones written in English and with clear description of treatment protocol, objective outcome measurements and proof that significant results could be attributed to use of NAM were included in this review. Studies relating to surgical, aesthetic, functional, socio-economical effects of NAM appliances and evolution of NAM appliances, especially CAD/CAM technologies were included.

\section{Exclusion criteria}

Articles that were excluded were related to the following reasons: presurgical infant orthopaedics (PSIO) different from NAM, small sample size ( $<10$ patients), long-term NAM efficiency reports (more than 18 months, usually after primary surgeries), bilateral clefts of the lip and/or palate and articles that lacked a control group.

\section{Sequential search strategy}

First, article titles were screened in order to exclude the impertinent studies. Then, abstracts were screened in order to exclude studies without inclusion criteria. Next, the selection of articles was performed after reviewing the "Material and Methods" and "Results" sections based on inclusion and exclusion criteria. The final stage of inclusion articles was based on evidence ratings according to the Oxford Center for Evidencebased Medicine-Level of Evidence (March 2009) [14].

\section{Data extraction}

Data extraction was performed with tables addressing different subjects: surgical, aesthetical, functional, 
socio-economical effects of NAM appliances and the evolution of NAM appliances, especially threedimensional technology. Each table includes: author, year of publication, type of study, type of cleft lip and/ or palate, aim of the study, sample size, presence or not of a control group, method assessment, effect of nasal molding, effect of alveolar molding, "other" outcomes, follow-up and study limit.

\section{Assessment of methodological quality}

Two independent researchers performed the literature search. Any disagreement regarding inclusion and exclusion criteria were discussed and resolved. Recommendations proposed in the general methods for Cochrane reviews in order to reduce the risk of bias assessment in studies were followed in the selection of articles [15].

\section{Statistical analysis}

Meta-analyses were to be conducted only if there were studies of similar comparison, reporting the same outcome measures. However, the studies included revealed considerable variations in design (i.e. large diversity of no-NAM presurgical appliances, methodology and landmark used to assess the outcomes of NAM compare to other appliances).

\section{RESULTS \\ Study selection and characteristics}

Out of 356 articles, 145 were further evaluated (Figure 1), 48 were considered relevant according to their abstract but 20 were excluded (Table 2). Twenty-eight articles were included and classified by category in tables (Table 3 and 4): "NAM impact on primary repair surgery", "Aesthetical and functional NAM outcomes", "Efficiency of NAM compared to other appliance without nasal stents", "NAM technique improvement", "Three-dimensional technology in NAM approach", "Socio-economical impact with NAM approach", "Complications with NAM treatment". In each category author, year of publication, type of study, type of cleft lip and/or palate, aim of the study, sample size, presence or not of a control group, method assessment, effect of nasal molding, effect of alveolar molding, "other" outcomes, follow-up and study limit could be extracted.

Four types of design study were found: randomized control trials, prospective, retrospective longitudinal studies and case series. Twenty studies were excluded because of their small sample size ( $<10$ cleft patients)
[16-21] or/and their follow-up exceeding 18 months [22-31]. Two studies relating to nasal and alveolar outcomes after PSIO treatment was excluded because the used appliance was not NAM [32,33]. Two studies were excluded because of lack of evidence $[34,35]$.

\section{Risk of bias within studies}

Each article has been ranked according to the Oxford Center for Evidence-based Medicine-Level [14] and at two external reviewers reviewed every manuscript.

\section{Statistical analysis}

No meta-analyses could not be performed due to the heterogeneity between the studies.

\section{NAM impact on primary repair surgeries}

The purpose of the PSIO is to facilitate primary repair of the lip and reduce the secondary revision. In the present review, three articles, using control groups, were selected to illustrate the benefit of NAM. In 1998, a retrospective review on 32 patients (18 cleft patients treated with NAM and 14 treated without NAM) by Santiago et al. [36] showed that NAM associated with gingivoperiosteoplasty (GGP) decreases the number of patients who require a secondary alveolar bone graft procedure. In a recent quasi-experimental study, surgeons from the American Cleft Palate-Craniofacial Association assessed 20 photographs of cleft lip and/or palate patients [37]. They were asked to evaluate the outcome after NAM treatment, and the likelihood of these patients requiring revision surgeries. They reported that NAM seems to reduce the necessity for over corrective surgeries. The advantage of the NAM treatment is confirmed with another recent study by Broder et al. [38]. It highlighted that caregivers reported better postsurgery outcomes in the NAM group compared with no-NAM group $(\mathrm{P}<0.05)$, particularly in relation to the appearance of the nose.

\section{Aesthetic and functional NAM outcomes}

NAM is composed of an alveolar plate and an attached nasal stent. The two parts of this device take advantage of the high plasticity of the skeletal maxillary alveolar bone and the neonatal nasal cartilage to induce an alveolar and a nasal positive remodelling. The main goal of NAM is to reduce the width of the gap between the alveolar segments. Nasal stents may be added to the palatal plate to improve the deformity of the nasal cartilages and reduce the nostril asymmetry. 


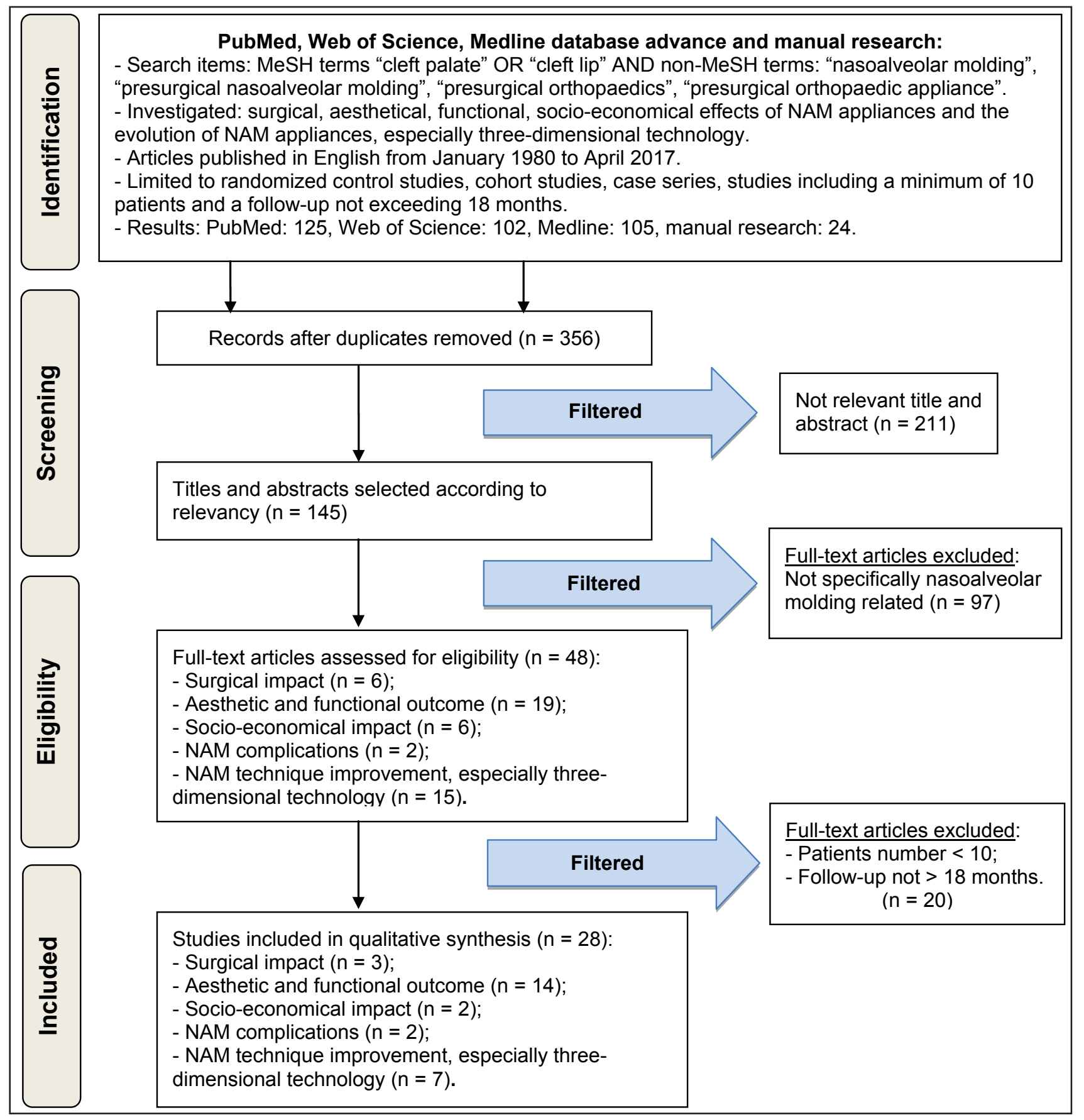

Figure 1. Flow diagram of studies selection according PRISMA guidelines.

Five prospective studies [39-43] and one retrospective study [44] compared PSIO with and without a nasal stent. All demonstrated that presurgical devices with a nasal component have the ability elongate the columella, and improve nasal asymmetry. Beside its nasal effect, three prospective studies showed that the nasal component has also a positive effect on the cleft defect [40-42]. Among the group of studies that assesses only the nasal molding, 2 studies were included. These reported an improvement of nasal symmetry and alar cartilage depression with NAM before primary surgeries $[\underline{45}, \underline{46}]$.

In the present review, four publications assessed nasal and alveolar effects. All showed that the alveolar gap, nasal width and nasal asymmetry were significantly reduced at the end of the PSIO therapy, prior to the primary lip repair [47-50]. Results presented by Shetty et al. [51], highlighted that a treatment started prior to one month of age has a greater impact on the nasal symmetry than treatments started after this period. In another study by Shetty et al. [52], are comparing the patients that initiate the treatment before 1 month, between 1 and 6 months and patients that started NAM after 6 months. It showed that beneficial NAM outcomes are increased if the treatment is started as soon as possible. 
Table 2. Excluded articles, classified by topic used for data extraction: surgical, aesthetical, functional, socio-economical effects of nasoalveolar molding (NAM) appliances, the improvements of NAM appliances and the three-dimensional technology (CAD/CAM)



$\mathrm{CAD} / \mathrm{CAM}=$ computer-aided design/computer-assisted manufacture; $\mathrm{PS}=$ prospective study; $\mathrm{RS}=$ retrospective study; $\mathrm{CS}$ = case series; UCLP = unilateral cleft lip and/or palate; $\mathrm{BCLP}=$ bilateral cleft lip and/or palate; $\mathrm{CLP}=$ cleft lip and/or palate; GPP = gingivoperiosteoplasty. 


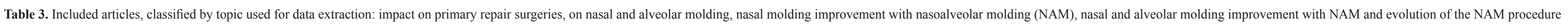

\begin{tabular}{|c|c|c|c|c|c|c|c|c|c|c|c|c|c|}
\hline Topic & Study & $\begin{array}{c}\text { Year of } \\
\text { publication }\end{array}$ & Study design & \begin{tabular}{|c|}
$\begin{array}{c}\text { Evidence } \\
\text { level }\end{array}$ \\
\end{tabular} & Cleft & Aim of the study & Sample size & Control group & Method assessment & Effect of nasal molding & Effect of alveolar molding & Other outcomes & Follow-up \\
\hline \multirow{3}{*}{$\begin{array}{c}\text { Impact on } \\
\text { primary repair } \\
\text { surgeries }\end{array}$} & $\begin{array}{c}\text { Santiago et al. } \\
{[36]}\end{array}$ & 1998 & $\begin{array}{l}\text { Retrospective blind } \\
\text { study }\end{array}$ & III & UCLP & $\begin{array}{l}\text { Compare the need of bone graft } \\
\text { between patients who undergo } \\
\text { NAM + GPP and no-NAM }\end{array}$ & $32(\mathrm{NAM}+\mathrm{GPP}: 18)$ & No-NAM: 14 & Clinical assessment & - & \begin{tabular}{|c|} 
NAM group: 8 required \\
bone graft; \\
no-NAM group: all required \\
bone graft
\end{tabular} & - & Before surgery \\
\hline & $\underset{\text { Rubin et al. }}{[37]}$ & 2015 & $\begin{array}{l}\text { Quasi-experimental } \\
\text { study }\end{array}$ & III & UCLP & $\begin{array}{c}\text { Assessment by the surgeon: } \\
\text { necessity of secondary nasal } \\
\text { revision surgery with and without } \\
\text { NAM }\end{array}$ & $\begin{array}{l}176 \text { of the } 731 \text { surgeons } \\
\text { accepted to answer the } \\
\text { survey- NAM: } 10 ; \\
\text { no-NAM: } 10\end{array}$ & No-NAM: 10 & $\begin{array}{c}\text { Two-dimensional } \\
\text { photographs }\end{array}$ & \begin{tabular}{|c|} 
Necessity secondary nasal revision \\
surgery: \\
NAM group: $3 \% ;$ \\
no-NAM group: $21 \%$. \\
Not statistically significant \\
\end{tabular} & - & $\begin{array}{l}\text { Patient with NAM cost } \\
\quad \$ 500 \text { less }\end{array}$ & Before the surgery \\
\hline & $\begin{array}{l}\text { Broder et al. } \\
\quad[38]\end{array}$ & 2016 & $\begin{array}{l}\text { Prospective non- } \\
\text { randomized study }\end{array}$ & II & $\begin{array}{l}\text { UCLP } \\
\text { BCLP }\end{array}$ & \begin{tabular}{|c|}
$\begin{array}{c}\text { Examines clinician and caregiver } \\
\text { appraisals of primary cleft lip and } \\
\text { nasal reconstruction }\end{array}$ \\
\end{tabular} & $\begin{array}{l}\text { NAM: } 62 ; \\
\text { No-NAM: } 48\end{array}$ & - & $\begin{array}{l}\text { Two-dimensional } \\
\text { photographs }\end{array}$ & $\begin{array}{c}\text { Better postsurgery outcomes in the } \\
\text { NAM group }\end{array}$ & - & - & 13 months \\
\hline \multirow{6}{*}{$\begin{array}{l}\text { Nasal stent } \\
\text { impact on } \\
\text { nasal and } \\
\text { alveolar } \\
\text { molding }\end{array}$} & $\begin{array}{l}\text { Punga and } \\
\text { Sharma [39] }\end{array}$ & 2013 & Prospective study & II & $\begin{array}{l}\text { UCLP } \\
\text { BCLP }\end{array}$ & $\begin{array}{c}\text { Comparison between treatment } \\
\text { with a presurgical appliance with } \\
\text { and without nasal stent }\end{array}$ & \begin{tabular}{|l|}
20 (with nasal stents: $10 ;$ \\
without nasal stents: 10$)$
\end{tabular} & ; & $\begin{array}{c}\text { Two-dimensional } \\
\text { photographs }\end{array}$ & $\begin{array}{c}\text { Increase the columella length with } \\
\text { a nasal stents }\end{array}$ & - & - & $\begin{array}{l}\text { Every } 2-3 \text { weeks } \\
\text { until lip repair }\end{array}$ \\
\hline & $\begin{array}{c}\begin{array}{c}\text { Monasterio et } \\
\text { al. [40] }\end{array} \\
\end{array}$ & 2013 & Prospective study & II & UCLP & $\begin{array}{c}\text { Compare two techniques: nasal } \\
\text { elevator and NAM-Grayson }\end{array}$ & $\begin{array}{c}40 \text { (NAM Grayson: } 20 ; \\
\text { nasal elevator: } 20 \text { ) }\end{array}$ & - & $\begin{array}{l}\text { Two-dimensional } \\
\text { photographs casts }\end{array}$ & \begin{tabular}{|c|} 
Two methods improved \\
significantly the nasal asymmetry \\
\end{tabular} & \begin{tabular}{|c|} 
Two methods reduced \\
significantly the cleft width
\end{tabular} & - & \begin{tabular}{|c|}
$\begin{array}{c}\text { months, (before the } \\
\text { surgery) }\end{array}$ \\
\end{tabular} \\
\hline & $\underset{[41]}{\text { Isogawa et al. }}$ & 2010 & Prospective study & II & UCLP & \begin{tabular}{|c|} 
Compare effect between Hotz \\
plate modified by adding a nasal \\
stent and modified NAM \\
\end{tabular} & 10 (NAM: 5; Hotz: 5) & - & Casts & $\begin{array}{l}\text { Favourable effect obtains with } \\
\text { PNAM }\end{array}$ & $\begin{array}{c}\text { Favourable effect with both } \\
\text { techniques }\end{array}$ & - & $\begin{array}{l}\text { Around } 130 \text { days } \\
\text { (4 months) }\end{array}$ \\
\hline & $\begin{array}{l}\text { Sasaki et al. } \\
{[42]}\end{array}$ & 2012 & Prospective study & II & UCLP & \begin{tabular}{|c|} 
Compare the effect between \\
appliance with NAM and passive \\
method with only action of \\
alveolar plate \\
\end{tabular} & $\begin{array}{l}28 \text { (NAM: } 13 ; \\
\text { Hotz plate: } 15 \text { ) }\end{array}$ & $\begin{array}{c}\text { Control with } \\
\text { the symmetric } \\
\text { nostril }\end{array}$ & $\begin{array}{l}\text { Two-dimensional } \\
\text { photographs casts }\end{array}$ & $\begin{array}{l}\text { Better naris morphology in the } \\
\text { NAM group }\end{array}$ & $\begin{array}{l}\text { Cleft gap smaller in the } \\
\text { NAM group }\end{array}$ & - & $\begin{array}{l}\text { Just before the } \\
\text { surgery and after }\end{array}$ \\
\hline & $\underset{[43]}{\text { Nakamura et al. }}$ & 2009 & Prospective study & II & UCLP & \begin{tabular}{|c|} 
Assess outcome nasal correction \\
after NAM, compare with Hotz \\
plate
\end{tabular} & 30 (NAM: 15; Hotz: 15) & & $\begin{array}{l}\text { Two-dimensional } \\
\text { photographs } \\
\text { photographs }\end{array}$ & $\begin{array}{l}\text { Better nasal shape in the NAM } \\
\text { group }\end{array}$ & - & - & $\begin{array}{l}1 \text { and } 5 \text { years post- } \\
\text { operative }\end{array}$ \\
\hline & Kozelj [44] & 2007 & Retrospective study & III & UCLP & \begin{tabular}{|c|}
$\begin{array}{c}\text { Compare presurical orthopaedic } \\
\text { without nasal stents and with } \\
\text { stents }\end{array}$ \\
\end{tabular} & $\begin{array}{c}\text { With nasal stents: } 16 ; \\
\text { without nasal stents: } 16\end{array}$ & & $\begin{array}{c}\text { Two-dimensional } \\
\text { photographs } \\
\text { photographs } \\
\end{array}$ & $\begin{array}{l}\text { Nose was more symmetric with } \\
\text { nasal stents }\end{array}$ & - & - & 1 year after lip repair \\
\hline \multirow{2}{*}{$\begin{array}{c}\text { Nasal molding } \\
\text { improvment } \\
\text { with NAM }\end{array}$} & \begin{tabular}{|c} 
López-Palacio \\
et al. [45]
\end{tabular} & 2012 & Prospective study & II & UCLP & Nasal improvement with NAM & 17 & $\begin{array}{c}\text { Non-cleft } \\
\text { nostril }\end{array}$ & \begin{tabular}{|c|} 
Two-dimensional \\
photographs of casts
\end{tabular} & $\begin{array}{l}\text { Improved of nasal tip projection, } \\
\text { alar cartilage depression and }\end{array}$ & - & - & $\begin{array}{l}\text { Before the primary } \\
\text { rhinocheiloplasty } \\
\text { (103 days), }\end{array}$ \\
\hline & $\begin{array}{c}\text { Gomez et al. } \\
{[46]}\end{array}$ & 2012 & Prospective study & II & UCLP & Nasal improvement with NAM & 30 & - & $\begin{array}{c}\text { Two-dimensional } \\
\text { photographs of casts }\end{array}$ & $\begin{array}{c}\text { Reduction of cleft columella } \\
\text { deviation, improved columella } \\
\text { length }\end{array}$ & - & - & $\begin{array}{l}\text { Before lip surgery } \\
\text { (146 days) }\end{array}$ \\
\hline \multirow{6}{*}{$\begin{array}{c}\text { Nasal and } \\
\text { alveolar } \\
\text { molding } \\
\text { improvment } \\
\text { with NAM }\end{array}$} & $\begin{array}{l}\text { Keçik and } \\
\text { Enacar [47] }\end{array}$ & 2009 & Prospective study & III & UCLP & $\begin{array}{c}\text { NAM effect on nasal and alveolar } \\
\text { tissues }\end{array}$ & 22 & - & $\begin{array}{l}\text { Two-dimensional } \\
\text { photographs scan } \\
\text { on cast }\end{array}$ & $\begin{array}{c}\text { Reduction alar base width and the } \\
\text { deviation of the columella }\end{array}$ & $\begin{array}{c}\begin{array}{c}\text { Reduction of the cleft width, } \\
\text { arch length }\end{array} \\
\text { a }\end{array}$ & - & $\begin{array}{l}6 \text { months (before } \\
\text { surgeries) }\end{array}$ \\
\hline & $\begin{array}{c}\text { Jaeger. et al. } \\
{[48]}\end{array}$ & 2007 & Prospective study & III & UCLP & $\begin{array}{c}\text { Evaluate nasal symmetry, gap } \\
\text { reduction }\end{array}$ & 11 & - & $\begin{array}{c}\begin{array}{c}\text { Two-dimensional } \\
\text { photographs }\end{array} \\
\end{array}$ & $\begin{array}{c}\text { Improvement nasal symmetry and } \\
\text { nostril shape }\end{array}$ & Cleft gap reduction & & $\begin{array}{c}\text { After NAM treatment } \\
\text { (max } 23 \text { weeks) }\end{array}$ \\
\hline & Pai et al. [49] & 2005 & Case-series & III & UCLP & $\begin{array}{c}\text { Evaluate nasal symmetry and } \\
\text { width }\end{array}$ & 57 & $\begin{array}{c}\text { Non affected } \\
\text { side }\end{array}$ & \begin{tabular}{|c|}
$\begin{array}{c}\text { Two-dimensional } \\
\text { photographs }\end{array}$ \\
\end{tabular} & $\begin{array}{c}\text { Effect on nasal symmetry, height, } \\
\text { and columella angle }\end{array}$ & - & $\begin{array}{l}\text { Relapse of nostril shape } \\
\text { in width }\end{array}$ & 1 year \\
\hline & $\begin{array}{c}\text { Ezzat et al. } \\
{[50]}\end{array}$ & 2007 & \begin{tabular}{|l}
$\begin{array}{l}\text { Prospective, blinded } \\
\text { measurement study }\end{array}$ \\
\end{tabular} & II & UCLP & \begin{tabular}{|c|} 
Evaluate improvement alveolar \\
cleft and nose symmetry
\end{tabular} & 12 & - & $\begin{array}{c}\text { Intra-oral and extra- } \\
\text { oral casts }\end{array}$ & Nasal symmetry improvement & Cleft gap reduction & - & Mean 110 days \\
\hline & $\begin{array}{c}\text { Shetty et al. } \\
{[51]}\end{array}$ & 2012 & Prospective study & II & UCLP & $\begin{array}{c}\text { Evaluation of the NAM treatment } \\
\text { depending on the moment when } \\
\text { the treatment is started }\end{array}$ & 45 & No-NAM: 15 & \begin{tabular}{|c|} 
Two-dimensional \\
photographs, dento- \\
facial impression \\
\end{tabular} & $\begin{array}{c}\text { Nasal measurements are improved } \\
\text { with NAM group }\end{array}$ & - & - & $\begin{array}{c}\text { Before NAM } \\
\text { treatment, before } \\
\text { surgery, at } 18 \text { months }\end{array}$ \\
\hline & $\begin{array}{l}\text { Shetty et al. } \\
{[52]}\end{array}$ & 2016 & Prospective study & II & UCLP & $\begin{array}{c}\text { To compare the effectiveness of } \\
\text { NAM in infants before and after } 6 \\
\text { months of age }\end{array}$ & $\begin{array}{l}150 \\
\text { (birth to } 1 \text { month: } 50 ; \\
1 \text { to } 6 \text { months: } 50 ; \\
6 \text { months to } 1 \text { year: } 50\end{array}$ & - & Cast landmark & $\begin{array}{c}\text { Nasal height, nasal dome height, } \\
\text { and columella height reduce with } \\
\text { NAM. }\end{array}$ & $\begin{array}{l}\text { Intersegment distance } \\
\text { reduced }\end{array}$ & $\begin{array}{l}\text { Patients who presented } \\
\text { for treatment before } 1 \\
\text { month of age benefited } \\
\text { the most }\end{array}$ & 1 year \\
\hline \multirow{2}{*}{$\begin{array}{l}\text { Evolution } \\
\text { of the NAM } \\
\text { procedure }\end{array}$} & $\begin{array}{l}\text { Chang et al. } \\
{[58]}\end{array}$ & 2014 & $\begin{array}{c}\text { Randomized } \\
\text { prospective, single } \\
\text { blind trial } \\
\end{array}$ & II & UCLP & $\begin{array}{l}\text { Compare traditional (Grayson) } \\
\text { NAM and modified (Figueroa) } \\
\text { NAM }\end{array}$ & $\begin{array}{l}30 \text { (Grayson NAM: } 15 \\
\text { Figueroa NAM: } 15 \text { ) }\end{array}$ & - & $\begin{array}{l}\text { Two-dimensional } \\
\text { photographs }\end{array}$ & $\begin{array}{l}\text { Similar results in term of nasal } \\
\text { result }\end{array}$ & - & - & $\begin{array}{l}\begin{array}{l}\text { Before surgery, one } \\
\text { week after surgery } \\
\text { and } 6 \text { month }\end{array} \\
\end{array}$ \\
\hline & Liao et al. [59] & 2012 & $\begin{array}{l}\text { Retrospective, blind } \\
\text { study }\end{array}$ & II & UCLP & $\begin{array}{c}\text { Compare traditional (Grayson) } \\
\text { NAM and modified (Figueroa) } \\
\text { NAM }\end{array}$ & $\begin{array}{l}\text { 63 (Grayson NAM: 31; } \\
\text { Figueroa NAM: 32) }\end{array}$ & - & $\begin{array}{l}\text { Two-dimensional } \\
\text { photographs }\end{array}$ & $\begin{array}{c}\text { Grayson NAM was more effective } \\
\text { to reduce nostril width but } \\
\text { required more corrections. }\end{array}$ & - & $\begin{array}{l}\text { More ulcerations with } \\
\text { Grayson NAM, }\end{array}$ & 15 weeks \\
\hline
\end{tabular}

$\mathrm{UCLP}=$ unilateral cleft lip and/or palate; BCLP = bilateral cleft lip and/or palate; PNAM = presurgical nasoalveolar molding; $\mathrm{n}=$ number of patients. 
Table 4. Included articles, articles concerning the three-dimensional technology (CAD-CAM)

\begin{tabular}{|c|c|c|c|c|c|c|c|c|c|c|c|c|c|c|}
\hline Topic & Study & $\begin{array}{c}\text { Year of } \\
\text { publication }\end{array}$ & $\begin{array}{l}\text { Study } \\
\text { design }\end{array}$ & $\begin{array}{c}\text { Evidence } \\
\text { level }\end{array}$ & Cleft & Aim of the study & $\begin{array}{l}\text { Sample } \\
\text { size }\end{array}$ & $\begin{array}{l}\text { Control } \\
\text { group }\end{array}$ & $\begin{array}{c}\text { Method } \\
\text { assessment }\end{array}$ & $\begin{array}{l}\text { Effect of nasal } \\
\text { molding }\end{array}$ & $\begin{array}{l}\text { Effect of } \\
\text { alveolar } \\
\text { molding }\end{array}$ & $\begin{array}{c}\text { Other } \\
\text { Outcomes }\end{array}$ & Follow-up & $\begin{array}{l}\text { Study } \\
\text { limit }\end{array}$ \\
\hline \multirow{3}{*}{$\begin{array}{l}\text { Three- } \\
\text { dimensional } \\
\text { analysis } \\
\text { (CAD) }\end{array}$} & $\begin{array}{c}\text { Singh et al. } \\
{[61]}\end{array}$ & 2007 & $\begin{array}{c}\text { Prospective } \\
\text { study }\end{array}$ & II & UCLP & $\begin{array}{c}\text { Evaluate with } \\
\text { three-dimensional } \\
\text { stereophotogrammetry } \\
\text { the facial morphology } \\
\text { after NAM }\end{array}$ & $\begin{array}{c}25 \\
\text { (NAM + } \\
\text { surgery: 15) }\end{array}$ & $\begin{array}{c}\text { No cleft: } \\
10\end{array}$ & \begin{tabular}{|c} 
Digital \\
stereophotogrammetry \\
used to capture three- \\
dimensional facial \\
image
\end{tabular} & $\begin{array}{c}\text { Three- } \\
\text { dimensional facial } \\
\text { morphology } \\
\text { virtually } \\
\text { indistinguishable } \\
\text { from the non-cleft }\end{array}$ & - & - & $\begin{array}{c}37 \text { weeks }+4 \\
\text { weeks after } \\
\text { surgery }\end{array}$ & - \\
\hline & $\begin{array}{l}\text { Baek and } \\
\text { Son [62] }\end{array}$ & 2006 & $\begin{array}{c}\text { Prospective } \\
\text { study }\end{array}$ & II & UCLP & $\begin{array}{l}\text { Assess alveolar } \\
\text { molding effect and } \\
\text { growth with CAD }\end{array}$ & 16 & NA & CAD & - & $\begin{array}{l}\text { Reduction } \\
\text { cleft gap }\end{array}$ & - & $\begin{array}{c}2 \text { months } \\
\text { after } \\
\text { cheilioplasty }\end{array}$ & $\begin{array}{l}\text { No } \\
\text { control } \\
\text { group }\end{array}$ \\
\hline & $\begin{array}{c}\text { Singh et al. } \\
{[63]}\end{array}$ & 2005 & $\begin{array}{c}\text { Prospective } \\
\text { study }\end{array}$ & III & UCLP & $\begin{array}{c}\text { Evaluate three- } \\
\text { dimensional change } \\
\text { nasal morphology } \\
\text { with NAM }\end{array}$ & 10 & NA & $\begin{array}{c}\text { Three-dimensional } \\
\text { facial image } \\
\text { captured with digital } \\
\text { stereophotogrammetry }\end{array}$ & $\begin{array}{c}\text { Size increase } \\
\text { on the cleft } \\
\text { and non-cleft } \\
\text { side. Symmetry } \\
\text { improved }\end{array}$ & - & - & $\begin{array}{l}\text { Before } \\
\text { surgical } \\
\text { repair }\end{array}$ & $\begin{array}{l}\text { No } \\
\text { control } \\
\text { group }\end{array}$ \\
\hline \multirow{2}{*}{$\begin{array}{c}\text { Three- } \\
\text { dimensional } \\
\text { analysis (CAD) } \\
\text { and printing } \\
\text { (CAM) }\end{array}$} & $\begin{array}{c}\text { Shen et al. } \\
\text { [53] }\end{array}$ & 2015 & $\begin{array}{c}\text { Prospective } \\
\text { study }\end{array}$ & II & UCLP & $\begin{array}{c}\text { Evaluate effect three- } \\
\text { dimensional printing } \\
\text { NAM }\end{array}$ & 17 & None & $\begin{array}{c}\text { Measured on three- } \\
\text { dimensional computed } \\
\text { tomography scans } \\
\text { before and after NAM } \\
\text { treatment }\end{array}$ & 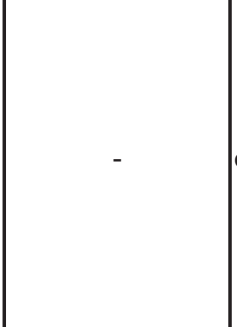 & $\begin{array}{l}\text { Alveolus } \\
\text { became more } \\
\text { contiguous and } \\
\text { cleft gap was } \\
\text { reduced }\end{array}$ & \begin{tabular}{|c}
11 patients \\
had mucosal \\
irritation, \\
minor \\
mucosal \\
ulceration, \\
decrease cost \\
of treatment
\end{tabular} & $\begin{array}{c}\text { Post } \\
\text { treatment } \\
\text { (before } \\
\text { cheiloplasty) }\end{array}$ & - \\
\hline & $\begin{array}{c}\text { Yu et al. } \\
{[64]}\end{array}$ & 2013 & $\begin{array}{c}\text { Randomized } \\
\text { control } \\
\text { study }\end{array}$ & II & UCLP & $\begin{array}{c}\text { Evaluate efficiency of } \\
\text { CAD-NAM therapy } \\
(7-10 \text { pairs of } \\
\text { appliances })\end{array}$ & $\begin{array}{c}30 \\
\text { (CAD-NAM } \\
\text { treated: } 15)\end{array}$ & $\begin{array}{c}\text { Non- } \\
\text { presurgical } \\
\text { therapy: } 15\end{array}$ & $\begin{array}{l}\text { Measurement on } \\
\text { scanned cast }\end{array}$ & - & $\begin{array}{l}\text { Reduce cleft } \\
\text { gap and } \\
\text { arch length. } \\
\text { Decrease } \\
\text { alveolar high }\end{array}$ & - & $\begin{array}{c}\text { Average } 123 \\
\text { days }\end{array}$ & - \\
\hline
\end{tabular}

$\mathrm{UCLP}=$ unilateral cleft lip and/or palate; $\mathrm{BCLP}=$ bilateral cleft lip and/or palate; $\mathrm{CAD} / \mathrm{CAM}=$ computer-aided design/computer-assisted manufacture. 


\section{Reduction of the cost of presurgical treatment}

Among the included studies, the one introducing three-dimensional technology report that NAM using three-dimensional technology has the potential to further decrease the cost of treatment by reducing the clinical chair time and the cost of NAM appliance $[\underline{53}, \underline{54}]$.

\section{Soft tissue, hard tissue and compliance complications}

Despite the non-invasive effect of NAM therapy, some complications during and after NAM treatment can occur. One publication relating to NAM complications was included in the present review: a retrospective research [55]. According to LevyBercowski et al. [55], 74\% of patients developed complications involving the soft tissue (mucosal ulceration, bleeding, tissue fungal infections and irritation); 7\% involving the hard tissue (asymmetrical configuration of the arch) and 39\% reported a lack of compliance. Among the soft and hard tissue complications, only ulcerations may be taken in account as a significant complication since the sample size is statistically small (27 patients). This suggests that improvements can be made to the traditional NAM appliance in order to reduce soft tissue problems [56].

\section{Previous evolution of Grayson technique}

Since the description of the NAM appliance by Grayson et al. []], several modifications have been proposed. Mitsuyoshi et al. [57] reported the first modification in 2004. They suggested the use of a nasal stent constructed in cobalt-chrome wire, which is believed to enhance manual control of the force and direction of the stent by the operator [57]. Bennun and Figueroa [35] provided another modification known as dynamic presurgical nasal remodelling. Two of the selected studies assessed this last modified technique and both concluded that the Figueroa appliance (with a large nasal stent) decreases the risk of ulceration compared to the traditional (Grayson) appliance $[\underline{58,59}]$.

However, all these techniques have the potential to irritate the soft tissues, are time consuming and can discourage patients and family. After taking the impression, NAM requires manual fabrication of the appliance. The region of the cleft is filled with wax, the cast is duplicated, the plate is fabricated with heat-cured acrylic resin, and finally the surface is polished [60]. Moreover, frequent visits are needed to adjust regularly the appliance by adding sequential selective resin. Finally, all these techniques do not follow a strict and repeatable protocol and the fabrication method is totally operator dependent. They may be considered as outdated compared to innovative three-dimensional technology used in other fields of dentistry, which have embraced digital technology.

\section{Emergence and benefit of three-dimensional analysis and printing}

Rapid prototyping technology was introduced in the 1990s to medicine and dentistry. Computer-aided design (CAD) made it possible to capture digital stereo-photogrammetry with laser scanning and provided high-accurate measurements from defined landmarks. Computer-aided manufacturing (CAM) allowed printing of a sequential set of appliances obtained from the treatment design software. Threedimensional technology for presurgical cleft treatment is widely adopted in the medical industry. Several studies have been conducted to assess this technology. Two types of studies were selected. One type assessed diagnostic efficiency with the help of CAD system, the other type evaluated the treatment effectiveness of the CAM system. Three prospective studies have been selected and all of them confirm that CAD systems are very reliable techniques to assess and quantify the nasal and alveolar improvement after presurgical treatment [61-63].

Among the studies that evaluated CAM system, one prospective study and one randomized control trial were selected $[\underline{53}, 64]$. Both showed that this method is a more efficient, more precise and more predictable technology. First, laser scanning and digital model construction simplify the NAM procedure by manufacturing a series of appliances at once, saving clinic time by reducing chair-side adjustment time. Secondly, the patient's parents are able to change the NAM appliance weekly and come to the treatment center less frequently. Third, the ability to visualize the procedure on a desktop or laptop computer can simplify communication between orthodontist and patient.

\section{DISCUSSION}

In the present review, the authors sought to answer two questions regarding the efficacy of NAM on nonsyndromic unilateral lip and/or cleft patients before primary surgeries. Does NAM appliance help presurgical orthopeadics users to better achieve cleft treatment goals, especially reduce primary 
surgical needs? And, how does the three-dimensional technology improve NAM therapy?

The first question addressed the benefits of NAM on the subsequent lip repair at approximately 18 months. A study published by Santiago et al. [36] demonstrated that patients undergoing NAM followed by GGP had a reduced need of further bone grafting procedure. This trend is confirmed by surgeons and caregivers' assessments when they found that NAM treatment improve UCLP outcomes. Excluded studies (follow-up exceeding 18 months) [22,23] reinforce these findings, particularly the retrospective study conducted by Patel et al. [24]. This study showed indeed that patients with complete UCLP and BCLP treated with NAM had a lower risk of early secondary nasal revisions compare to patient without NAM. Subsequently this phenomenon explained the reduction of the cost of care with NAM treatment compare to no-NAM treatment.

This question was also related to the aesthetic and functional success of the NAM treatment. Prior studies noted that NAM significantly improves the shape of the nose and reduces the alveolar clef just before the primary repair surgeries [38-51]. Despite these positive reports, alveolar molding is still not universally utilized, and this situation is not always due to inexperience or unavailability but to scepticism regarding its efficacy. Several studies [65-70] have showed no significant effect of NAM in long-term follow-up (between 2 to 10 years old). Critical review of these has determined them to be high quality (prospective in design, large sample size, and long term follow-up). They have assessed different appliances: Latham-Millard appliance $[\underline{71}, \underline{72}]$, other active appliance [6ㅜ $]$ and passive presurgical orthopaedics [66-68]. The randomized Dutchcleft series conducted in 2004 [73], 2006 [67], 2008 [74], 2009 [68] and 2015 [69,70] are some of the most cited. However, none of these latter studies assessed NAM effect in particular. Only one study, conducted by Clark et al. [75] challenged the effect of NAM by demonstrating that longterm alveolar improvement after NAM treatment was not confirmed with the three-dimensional measurements.

Another issue related to the first question is the socioeconomical impact of NAM therapy because although successful treatment of the aesthetic and functional aspects of orofacial cleft anomalies is possible, it is still technically challenging, lengthy and costly. Therefore, it is important to take into consideration the economic impact of the NAM on the overall cost of the therapy and the satisfaction of parents during the presurgical orthopaedic treatment. Among included studies, Shen et al. [53] and Chen et al. [54] evaluated the cost of cleft treatment and demonstrated that the NAM appliance has the potential to decrease the overall cost of cleft treatment. Two excluded retrospective studies confirmed this trend [30,31], NAM appeared indeed as a great mean to reduce the complexity, the number of the repair surgeries and subsequently the cost of care. One of them published by Shay et al. in 2015 [31], gives interesting results and showed that the cost of a group of patients treated with NAM (mean costs $\$ 3550.24 \pm \$ 667.27$ ) is significantly lower compare to a group of patients who received a surgical clef lip adhesion without NAM (mean costs $\$ 9370.55 \pm \$ 1691.79$ ). No study related to parent's cooperation during NAM therapy was included. If parental compliance during NAM therapy may be compared to other presurgical orthopaedics, it is important to mention a relevant Dutchcleft prospective randomized controlled trial conducted by Prahl et al. [33]. This study compares the acceptance of presurgical orthopaedics by the mother. An analysis of questionnaires filled out by the mothers of cleft patients at 6 weeks, 24 weeks and 58 weeks showed that families were motivated to follow the orthopaedic treatment despite the increased care time required [33]. A small descriptive study by Hopkins et al. [34] that captures on parent's lived experiences, highlights that education and providing support can substantially improve NAM treatment.

A final issue that needed to be addressed was the incidence of complications that occur during NAM treatment. Two publications highlighted different sets of complications related to use of the traditional NAM appliance (vomiting, ulceration, and non-compliance). Levy-Bercowski et al. [55] offer some preventive measures to facilitate NAM fabrication and reduce complications. These include specific techniques in taking the impression, limiting the posterior limit of the device, and minimizing the thickness of the occlusal plate (2 to $3 \mathrm{~mm}$ ) [55, $\underline{76}]$.

The second question the authors sought to answer was: does the three-dimensional technology improve NAM therapy? Three-dimensional technology is widely used in medicine [77], especially in maxillofacial surgery. $\mathrm{CAD} / \mathrm{CAM}$ is able to fabricate a reproducible, accurate and individual appliance in a short period of time, which suits the goal of individualized medicine where each patient requires a specific therapeutic approach using predictive simulation systems [78]. It is now possible to produce sequential NAM devices with a three-dimensional printer from stereolithographic files obtained from predictive simulation of cleft segment manipulation. 
According to Shen et al. [53], CAD [62,63] and CAM $[19,53]$, results in a more efficient and predictable NAM treatment. Other advantages are the potential decrease in cost and time for fabrication. This technology is also more reliable and it allows for visualisation of the treatment objective, assessment of the improvement and adaptation of the appliance). Three-dimensional technology is already widely used to treat cleft deformities. A preliminary prospective study by Ritschl et al. [20] is comparing the efficiency and presence of complications between NAM made manually $(n=6)$ and NAM produced with threedimensional technology $(\mathrm{n}=6)$ highlighted no difference between these two methods. However; the small sample size prevents any conclusion. Prospective studies with a large sample size should be conducted to assess the real potential of threedimensional-printed NAM devices. This innovative solution could address the shortcomings of NAM therapy and insure that NAM therapy becomes an integral part of the standard of care for unilateral cleft palate treatment.

\section{CONCLUSIONS}

According to the literature review, nasoalveolar molding appliance therapy offer positive surgical, aesthetical, functional and socio-economical effects on unilateral clefts of the lip and/or palate treatment when performed prior to primary repair surgeries. Three-dimensional technology seems to result in a more efficient and predictable nasoalveolar molding treatment. However, scientific evidence is lacking regarding the short-term effect of nasoalveolar molding appliances and the three-dimensional technology.

\section{ACKNOWLEDGMENTS AND DISCLOSURE STATEMENTS}

The authors do not have any financial interests, either directly or indirectly, in the products or information listed in the paper.

\section{REFERENCES}

1. Fisher MD, Fisher DM, Marcus JR. Correction of the cleft nasal deformity: from infancy to maturity. Clin Plast Surg. 2014 Apr;41(2):283-99. [Medline: 24607195] [doi: 10.1016/j.cps.2014.01.002]

2. Kirschner RE, LaRossa D. Cleft lip and palate. Otolaryngol Clin North Am. 2000 Dec;33(6):1191-215, v-vi. [Medline: 11449783] [doi: 10.1016/S0030-6665(05)70277-2]

3. McNeil CK. Orthodontic procedures in the treatment of congenital cleft palate. Dent Rec (London). 1950 May;70(5): 126-32. [Medline: 24537837]

4. McNeil CK. Oral and facial deformity. London: Pitman and Sons; 1954. p. 14-25.

5. Matsuo K, Hirose T. Nonsurgical correction of cleft lip nasal deformity in the early neonate. Ann Acad Med Singapore. 1988 Jul;17(3):358-65. [Medline: $\underline{3064700]}$

6. Grayson BH, Santiago PE, Brecht LE, Cutting CB. Presurgical nasoalveolar molding in infants with cleft lip and palate. Cleft Palate Craniofac J. 1999 Nov;36(6):486-98. [Medline: 10574667] [doi: 10.1597/1545-1569(1999)0362.3.CO;2]

7. Grayson BH, Cutting CB. Presurgical nasoalveolar orthopedic molding in primary correction of the nose, lip, and alveolus of infants born with unilateral and bilateral clefts. Cleft Palate Craniofac J. 2001 May;38(3):193-8. [Medline: 11386426] [doi: 10.1597/1545-1569(2001)0382.0.CO;2]

8. Henry C, Samson T, Mackay D. Evidence-based medicine: The cleft lip nasal deformity. Plast Reconstr Surg. 2014 May;133(5):1276-88. [Medline: 24776558]

9. van der Heijden P, Dijkstra PU, Stellingsma C, van der Laan BF, Korsten-Meijer AG, Goorhuis-Brouwer SM. Limited evidence for the effect of presurgical nasoalveolar molding in unilateral cleft on nasal symmetry: a call for unified research. Plast Reconstr Surg. 2013 Jan;131(1):62e-71e. [Medline: 23271555] [doi: 10.1097/PRS.0b013e318267d4a5]

10. Grayson BH. Discussion: Limited evidence for the effect of presurgical nasoalveolar molding in unilateral cleft on nasal symmetry: a call for unified research. Plast Reconstr Surg. 2013 Jan;131(1):75e-6e. [Medline: 23271557] [doi: 10.1097/PRS.0b013e318278cde6]

11. Abbott MM, Meara JG. Nasoalveolar molding in cleft care: is it efficacious? Plast Reconstr Surg. 2012 Sep;130(3): 659-66. [Medline: 22929251] [doi: 10.1097/PRS.0b013e31825dc10a]

12. Chang CS, Liao YF, Wallace CG, Chan FC, Liou EJ, Chen PK, Noordhoff MS. Long-term comparison of the results of four techniques used for bilateral cleft nose repair: a single surgeon's experience. Plast Reconstr Surg. 2014 Dec;134(6): 926e-36e. [Medline: 25415115] [doi: 10.1097/PRS.0000000000000715]

13. Moher D, Shamseer L, Clarke M, Ghersi D, Liberati A, Petticrew M, Shekelle P, Stewart LA; PRISMA-P Group. Preferred reporting items for systematic review and meta-analysis protocols (PRISMA-P) 2015 statement. Syst Rev. 2015 Jan 1;4:1. [Medline: 25554246] [PMC free article: 4320440] [doi: 10.1186/2046-4053-4-1] 
14. Al-Harbi K, Farrokhyar F, Mulla S, Fitzgerald P. Classification and appraisal of the level of clinical evidence of publications from the Canadian Association of Pediatric Surgeons for the past 10 years. J Pediatr Surg. 2009 May;44(5):1013-7. [Medline: 19433189] [doi: 10.1016/j.jpedsurg.2009.01.048]

15. Higgins JPT, Green S. Cochrane Handbook for Systematic Reviews of Interventions Version 5.1.0 (updated March 2011). The Cochrane Collaboration, 2011. [URL: http://handbook.cochrane.org]

16. Koya S, Shetty S, Husain A, Khader M. Presurgical Nasoalveolar Molding Therapy Using Figueroa's NAM Technique in Unilateral Cleft Lip and Palate Patients: A Preliminary Study. J Clin Pediatr Dent. 2016;40(5):410-6. [Medline: 27617383] [doi: 10.17796/1053-4628-40.5.410]

17. Simanca E, Morris D, Zhao L, Reisberg D, Viana G. Measuring progressive soft tissue change with nasoalveolar molding using a three-dimensional system. J Craniofac Surg. 2011 Sep;22(5):1622-5. [Medline: 21959400] [doi: 10.1097/SCS.0b013e31822e8ca0]

18. Braumann B, Keilig L, Bourauel C, Niederhagen B, Jäger A. 3-dimensional analysis of cleft palate casts. Ann Anat. 1999 Jan;181(1):95-8. [Medline: 10081569] [doi: 10.1016/S0940-9602(99)80102-2]

19. Yu Q, Gong X, Wang GM, Yu ZY, Qian YF, Shen G. A novel technique for presurgical nasoalveolar molding using computer-aided reverse engineering and rapid prototyping. J Craniofac Surg. 2011 Jan;22(1):142-6. [Medline: 21187760] [doi: 10.1097/SCS.0b013e3181f6f9ae]

20. Ritschl LM, Rau A, Güll FD, diBora B, Wolff KD, Schönberger M, Bauer FX, Wintermantel E, Loeffelbein DJ. Pitfalls and solutions in virtual design of nasoalveolar molding plates by using CAD/CAM technology--A preliminary clinical study. J Craniomaxillofac Surg. 2016 Apr;44(4):453-9. [Medline: 26880010] [doi: 10.1016/j.jcms.2016.01.008]

21. Loeffelbein DJ, Ritschl LM, Rau A, Wolff KD, Barbarino M, Pfeifer S, Schönberger M, Wintermantel E. Analysis of computer-aided techniques for virtual planning in nasoalveolar moulding. Br J Oral Maxillofac Surg. 2015 May;53(5):45560. [Medline: 25836048] [doi: 10.1016/j.bjoms.2015.03.002]

22. Hsieh $\mathrm{CH}$, Ko EW, Chen PK, Huang CS. The effect of gingivoperiosteoplasty on facial growth in patients with complete unilateral cleft lip and palate. Cleft Palate Craniofac J. 2010 Sep;47(5):439-46. [Medline: 20180706] [doi: 10.1597/08-207]

23. Dec W, Shetye PR, Davidson EH, Grayson BH, Brecht LE, Cutting CB, Warren SM. Presurgical nasoalveolar molding and primary gingivoperiosteoplasty reduce the need for bone grafting in patients with bilateral clefts. J Craniofac Surg. 2013 Jan;24(1):186-90. [Medline: 23348282] [doi: 10.1097/SCS.0b013e318270fd21]

24. Patel PA, Rubin MS, Clouston S, Lalezaradeh F, Brecht LE, Cutting CB, Shetye PR, Warren SM, Grayson BH. Comparative Study of Early Secondary Nasal Revisions and Costs in Patients With Clefts Treated With and Without Nasoalveolar Molding. J Craniofac Surg. 2015 Jun;26(4):1229-33. [Medline: 26080163] [doi: 10.1097/SCS.0000000000001729]

25. Maull DJ, Grayson BH, Cutting CB, Brecht LL, Bookstein FL, Khorrambadi D, Webb JA, Hurwitz DJ. Long-term effects of nasoalveolar molding on three-dimensional nasal shape in unilateral clefts. Cleft Palate Craniofac J. 1999 Sep;36(5):391-7. [Medline: 10499400] [doi: 10.1597/1545-1569(1999)0362.3.CO;2]

26. Chang CS, Por YC, Liou EJ, Chang CJ, Chen PK, Noordhoff MS. Long-term comparison of four techniques for obtaining nasal symmetry in unilateral complete cleft lip patients: a single surgeon's experience. Plast Reconstr Surg. 2010 Oct;126(4):1276-84. [Medline: 20885247] [doi: 10.1097/PRS.0b013e3181ec21e4]

27. Liou EJ, Subramanian M, Chen PK, Huang CS. The progressive changes of nasal symmetry and growth after nasoalveolar molding: a three-year follow-up study. Plast Reconstr Surg. 2004 Sep 15;114(4):858-64. [Medline: 15468390] [doi: 10.1097/01.PRS.0000133027.04252.7A]

28. Barillas I, Dec W, Warren SM, Cutting CB, Grayson BH. Nasoalveolar molding improves long-term nasal symmetry in complete unilateral cleft lip-cleft palate patients. Plast Reconstr Surg. 2009 Mar;123(3):1002-6. [Medline: 19319066] [doi: 10.1097/PRS.0b013e318199f46e]

29. Bennun RD, Perandones C, Sepliarsky VA, Chantiri SN, Aguirre MI, Dogliotti PL. Nonsurgical correction of nasal deformity in unilateral complete cleft lip: a 6-year follow-up. Plast Reconstr Surg. 1999 Sep;104(3):616-30. [Medline: 10456510] 10.1097/00006534-199909010-00002]

30. Pfeifer TM, Grayson BH, Cutting CB. Nasoalveolar molding and gingivoperiosteoplasty versus alveolar bone graft: an outcome analysis of costs in the treatment of unilateral cleft alveolus. Cleft Palate Craniofac J. 2002 Jan;39(1):26-9. [Medline: 11772166 ] [doi: 10.1597/1545-1569(2002)0392.0.CO;2]

31. Shay PL, Goldstein JA, Paliga JT, Wink J, Jackson OA, Low D, Bartlett SP, Taylor JA. A Comparative Cost Analysis of Cleft Lip Adhesion and Nasoalveolar Molding before Formal Cleft Lip Repair. Plast Reconstr Surg. 2015 Dec;136(6): 1264-71.. [Medline: 26595019] [doi: 10.1097/PRS.0b013e31829b69fe]

32. Yamada T, Mori Y, Mishima K, Sugahara T. Nasolabial and alveolar morphology following presurgical orthopaedic treatment in complete unilateral clefts of lip, alveolus and palate. J Craniomaxillofac Surg. 2003 Dec;31(6):343-7. [Medline: 14637062] [doi: 10.1016/j.jcms.2003.07.004]

33. Prahl C, Prahl-Andersen B, Van't Hof MA, Kuijpers-Jagtman AM. Presurgical orthopedics and satisfaction in motherhood: a randomized clinical trial (Dutchcleft). Cleft Palate Craniofac J. 2008 May;45(3):284-8.. [Medline: 18452361] [doi: $10.1597 / 07-045.1]$ 
34. Hopkins EE, Gazza E, Marazita ML. Parental experience caring for cleft lip and palate infants with nasoalveolar moulding. J Adv Nurs. 2016 Oct;72(10):2413-22. [Medline: 27144651] [doi: 10.1111/jan.12994]

35. Bennun RD, Figueroa AA. Dynamic presurgical nasal remodeling in patients with unilateral and bilateral cleft lip and palate: modification to the original technique. Cleft Palate Craniofac J. 2006 Nov;43(6):639-48. [Medline: 17105322] [doi: 10.1597/05-054]

36. Santiago PE, Grayson BH, Cutting CB, Gianoutsos MP, Brecht LE, Kwon SM. Reduced need for alveolar bone grafting by presurgical orthopedics and primary gingivoperiosteoplasty. Cleft Palate Craniofac J. 1998 Jan;35(1):77-80. [Medline: 9482227] [doi: 10.1597/1545-1569(1998)0352.3.CO;2]

37. Rubin MS, Clouston S, Ahmed MM, M Lowe K, Shetye PR, Broder HL, Warren SM, Grayson BH. Assessment of presurgical clefts and predicted surgical outcome in patients treated with and without nasoalveolar molding. J Craniofac Surg. 2015 Jan;26(1):71-5. [Medline: 25534051] [doi: 10.1097/SCS.0000000000001233]

38. Broder HL, Flores RL, Clouston S, Kirschner RE, Garfinkle JS, Sischo L, Phillips C. Surgeon's and Caregivers' Appraisals of Primary Cleft Lip Treatment with and without Nasoalveolar Molding: A Prospective Multicenter Pilot Study. Plast Reconstr Surg. 2016 Mar;137(3):938-45. [Medline: 26910677] [doi: 10.1097/01.prs.0000479979.83169.57]

39. Punga R, Sharma SM. Presurgical orthopaedic nasoalveolar molding in cleft lip and palate infants: a comparative evaluation of cases done with and without nasal stents. J Maxillofac Oral Surg. 2013 Sep;12(3):273-88. [Medline: 24431854] [doi: 10.1007/s12663-012-0434-y]

40. Monasterio L, Ford A, Gutiérrez C, Tastets ME, García J. Comparative study of nasoalveolar molding methods: nasal elevator plus DynaCleft ${ }^{\circledR}$ versus NAM-Grayson in patients with complete unilateral cleft lip and palate. Cleft Palate Craniofac J. 2013 Sep;50(5):548-54. [Medline: 22906392] [doi: 10.1597/11-245]

41. Isogawa N, Ochiai S, Mito T, Kindaichi J, Ishibashi N, Takagi Y, Ishikawa M. Three-Dimensional Comparison in Palatal Forms Between Modified Presurgical Nasoalveolar Molding Plate and Hotz's Plate Applied to the Infants With Unilateral Cleft Lip and Palate. Singapore Dent J. 2010 Jun;31(1):36-42. [Medline: 23739256] [doi: 10.1016/S0377-5291(12)70008-1]

42. Sasaki H, Togashi S, Karube R, Yanagawa T, Nakane S, Tabuchi K, Ishibashi N, Shinya Y, Ito H, Yamagata K, Onizawa K, Adachi K, Sekido M, Bukawa H. Presurgical nasoalveolar molding orthopedic treatment improves the outcome of primary cheiloplasty of unilateral complete cleft lip and palate, as assessed by naris morphology and cleft gap. J Craniofac Surg. 2012 Nov;23(6):1596-601. [Medline: 23147280] [doi: 10.1097/SCS.0b013e31825196dc]

43. Nakamura N, Sasaguri M, Nozoe E, Nishihara K, Hasegawa H, Nakamura S. Postoperative nasal forms after presurgical nasoalveolar molding followed by medial-upward advancement of nasolabial components with vestibular expansion for children with unilateral complete cleft lip and palate. J Oral Maxillofac Surg. 2009 Oct;67(10):2222-31. [Medline: 19761917] [doi: 10.1016/j.joms.2009.04.098]

44. Kozelj V. Experience with presurgical nasal molding in infants with cleft lip and nose deformity. Plast Reconstr Surg. 2007 Sep;120(3):738-45. [Medline: 17700126] [doi: 10.1097/01.prs.0000270847.12427.25]

45. López-Palacio AM, Cerón-Zapata AM, Gómez DF, Dávila-Calle AP, Ojalvo-Arias MA. Nasal changes with nasoalveolar molding in Colombian patients with unilateral cleft lip and palate. Pediatr Dent. 2012 May-Jun;34(3):239-44. [Medline: 22795158]

46. Gomez DF, Donohue ST, Figueroa AA, Polley JW. Nasal changes after presurgical nasoalveolar molding (PNAM) in the unilateral cleft lip nose. Cleft Palate Craniofac J. 2012 Nov;49(6):689-700. [Medline: 21846257] [doi: 10.1597/11-007]

47. Keçik D, Enacar A. Effects of nasoalveolar molding therapy on nasal and alveolar morphology in unilateral cleft lip and palate. J Craniofac Surg. 2009 Nov;20(6):2075-80. [Medline: 19881365] [doi: 10.1097/SCS.0b013e3181be88cf]

48. Jaeger M, Braga-Silva J, Gehlen D, Sato Y, Zuker R, Fisher D. Correction of the alveolar gap and nostril deformity by presurgical passive orthodontia in the unilateral cleft lip. Ann Plast Surg. 2007 Nov;59(5):489-94. [Medline: 17992140] [doi: 10.1097/01.sap.0000259001.98869.d8]

49. Pai BC, Ko EW, Huang CS, Liou EJ. Symmetry of the nose after presurgical nasoalveolar molding in infants with unilateral cleft lip and palate: a preliminary study. Cleft Palate Craniofac J. 2005 Nov;42(6):658-63. [Medline: 16241178] [doi: 10.1597/04-126.1]

50. Ezzat CF, Chavarria C, Teichgraeber JF, Chen JW, Stratmann RG, Gateno J, Xia JJ. Presurgical nasoalveolar molding therapy for the treatment of unilateral cleft lip and palate: a preliminary study. Cleft Palate Craniofac J. 2007 Jan;44(1): 8-12. [Medline: 17214541] [doi: 10.1597/06-009]

51. Shetty V, Vyas HJ, Sharma SM, Sailer HF. A comparison of results using nasoalveolar moulding in cleft infants treated within 1 month of life versus those treated after this period: development of a new protocol. Int J Oral Maxillofac Surg. 2012 Jan;41(1):28-36. [Medline: 22018821] [doi: 10.1016/j.ijom.2011.09.002]

52. Shetty V, Thakral A, Sreekumar C. Comparison of Early Onset Nasoalveolar Molding With Patients Who Presented for Molding Up to 1 Year of Age. J Oral Maxillofac Surg. 2016 Apr;74(4):811-27. [Medline: 26341679] [doi: 10.1016/j.joms.2015.08.004]

53. Shen C, Yao CA, Magee W 3rd, Chai G, Zhang Y. Presurgical nasoalveolar molding for cleft lip and palate: the application of digitally designed molds. Plast Reconstr Surg. 2015 Jun;135(6):1007e-1015e. [Medline: 26017607] [doi: 10.1097/PRS.0000000000001286] 
54. Chen YF, Liao YF. A modified nasoalveolar molding technique for correction of unilateral cleft nose deformity. J Craniomaxillofac Surg. 2015 Dec;43(10):2100-5. [Medline: 26541749] [doi: 10.1016/j.jcms.2015.10.003]

55. Levy-Bercowski D, Abreu A, DeLeon E, Looney S, Stockstill J, Weiler M, Santiago PE. Complications and solutions in presurgical nasoalveolar molding therapy. Cleft Palate Craniofac J. 2009 Sep;46(5):521-8. [Medline: 19929090] [doi: $10.1597 / 07-236.1]$

56. Rau A, Ritschl LM, Mücke T, Wolff KD, Loeffelbein DJ. Nasoalveolar molding in cleft care--experience in 40 patients from a single centre in Germany. PLoS One. 2015 Mar 3;10(3):e0118103. [Medline: 25734535] [PMC free article: 4347986] [doi: 10.1371/journal.pone.0118103]

57. Mitsuyoshi I, Masahiko W, Masayuki F. Simple modified preoperative nasoalveolar moulding in infants with unilateral cleft lip and palate. Br J Oral Maxillofac Surg. 2004 Dec;42(6):578-80. [Medline: 15544893] [doi: 10.1016/j.bjoms.2004.06.002]

58. Chang CS, Wallace CG, Pai BC, Chiu YT, Hsieh YJ, Chen IJ, Liao YF, Liou EJ, Chen PK. Comparison of two nasoalveolar molding techniques in unilateral complete cleft lip patients: a randomized, prospective, single-blind trial to compare nasal outcomes. Plast Reconstr Surg. 2014 Aug;134(2):275-82. [Medline: 24732649] [doi: 10.1097/PRS.0000000000000361]

59. Liao YF, Hsieh YJ, Chen IJ, Ko WC, Chen PK. Comparative outcomes of two nasoalveolar molding techniques for unilateral cleft nose deformity. Plast Reconstr Surg. 2012 Dec;130(6):1289-95. [Medline: 23190811] [doi: 10.1097/PRS.0b013e31826d16f3]

60. Kamble VD, Parkhedkar RD, Sarin SP, Patil PG, Kothari B. Simplifying cleft surgery by presurgical nasoalveolar molding (PNAM) for infant born with unilateral cleft lip, alveolus, and palate: a clinical report. J Prosthodont Res. 2013 Jul;57(3):224-31. [Medline: 23773376] [doi: 10.1016/j.jpor.2013.03.002]

61. Singh GD, Levy-Bercowski D, Yáñez MA, Santiago PE. Three-dimensional facial morphology following surgical repair of unilateral cleft lip and palate in patients after nasoalveolar molding. Orthod Craniofac Res. 2007 Aug;10(3):161-6. [Medline: 17651132] [doi: 10.1111/j.1601-6343.2007.00390.x]

62. Baek SH, Son WS. Difference in alveolar molding effect and growth in the cleft segments: 3-dimensional analysis of unilateral cleft lip and palate patients. Oral Surg Oral Med Oral Pathol Oral Radiol Endod. 2006 Aug;102(2):160-8. [Medline: 16876057 ] [doi: $10.1016 /$ i.tripleo.2005.09.009]

63. Singh GD, Levy-Bercowski D, Santiago PE. Three-dimensional nasal changes following nasoalveolar molding in patients with unilateral cleft lip and palate: geometric morphometrics. Cleft Palate Craniofac J. 2005 Jul;42(4):403-9. [Medline: 16001922] 10.1597/04-063.1]

64. Yu Q, Gong X, Shen G. CAD presurgical nasoalveolar molding effects on the maxillary morphology in infants with UCLP. Oral Surg Oral Med Oral Pathol Oral Radiol. 2013 Oct;116(4):418-26. [Medline: 24035109] [doi: 10.1016/j.0000.2013.06.032]

65. Adali N, Mars M, Petrie A, Noar J, Sommerlad B. Presurgical orthopedics has no effect on archform in unilateral cleft lip and palate. Cleft Palate Craniofac J. 2012 Jan;49(1):5-13. [Medline: 21823827] [doi: 10.1597/11-030]

66. Flinn W, Long RE, Garattini G, Semb G. A multicenter outcomes assessment of five-year-old patients with unilateral cleft lip and palate. Cleft Palate Craniofac J. 2006 May;43(3):253-8. [Medline: 16681397] [doi: 10.1597/04-093R.1]

67. Bongaarts CA, van 't Hof MA, Prahl-Andersen B, Dirks IV, Kuijpers-Jagtman AM. Infant orthopedics has no effect on maxillary arch dimensions in the deciduous dentition of children with complete unilateral cleft lip and palate (Dutchcleft). Cleft Palate Craniofac J. 2006 Nov;43(6):665-72. [Medline: 17105327] [doi: 10.1597/05-129]

68. Bongaarts CA, Prahl-Andersen B, Bronkhorst EM, Prahl C, Ongkosuwito EM, Borstlap WA, Kuijpers-Jagtman AM. Infant orthopedics and facial growth in complete unilateral cleft lip and palate until six years of age (Dutchcleft). Cleft Palate Craniofac J. 2009 Nov;46(6):654-63. [Medline: 19860495] [doi: 10.1597/08-034.1]

69. Noverraz RL, Disse MA, Ongkosuwito EM, Kuijpers-Jagtman AM, Prahl C. Transverse dental arch relationship at 9 and 12 years in children with unilateral cleft lip and palate treated with infant orthopedics: a randomized clinical trial (DUTCHCLEFT). Clin Oral Investig. 2015 Dec;19(9):2255-65. [Medline: 25855466] [doi: 10.1007/s00784-015-1451-2]

70. Noverraz RL, Disse MA, Ongkosuwito EM, Kuijpers-Jagtman AM, Prahl C. Erratum to: Transverse dental arch relationship at 9 and 12 years in children with unilateral cleft lip and palate treated with infant orthopedics: a randomized clinical trial (DUTCHCLEFT). Clin Oral Investig. 2015 Dec;19(9):2351. [Medline: 26275665] [doi: $10.1007 / \mathrm{s} 00784-015-1529-\mathrm{x}$ ]

71. Millard DR, Latham R, Huifen X, Spiro S, Morovic C. Cleft lip and palate treated by presurgical orthopedics, gingivoperiosteoplasty, and lip adhesion (POPLA) compared with previous lip adhesion method: a preliminary study of serial dental casts. Plast Reconstr Surg. 1999 May;103(6):1630-44. [Medline: 10323695] [doi: 10.1097/00006534-199905000-00009]

72. Berkowitz S, Mejia M, Bystrik A. A comparison of the effects of the Latham-Millard procedure with those of a conservative treatment approach for dental occlusion and facial aesthetics in unilateral and bilateral complete cleft lip and palate: part I. Dental occlusion. Plast Reconstr Surg. 2004 Jan;113(1):1-18. [Medline: 14707617] [doi: 10.1097/01.PRS.0000096710.08123.93]

73. Bongaarts CA, Kuijpers-Jagtman AM, van 't Hof MA, Prahl-Andersen B. The effect of infant orthopedics on the occlusion of the deciduous dentition in children with complete unilateral cleft lip and palate (Dutchcleft). Cleft Palate Craniofac J. 2004 Nov;41(6):633-41. [Medline: 15516167] [doi: 10.1597/03-051.1] 
74. Bongaarts CA, Prahl-Andersen B, Bronkhorst EM, Spauwen PH, Mulder JW, Vaandrager JM, Kuijpers-Jagtman AM. Effect of infant orthopedics on facial appearance of toddlers with complete unilateral cleft lip and palate (Dutchcleft). Cleft Palate Craniofac J. 2008 Jul;45(4):407-13. [Medline: 18616367] [doi: 10.1597/07-043.1]

75. Clark SL, Teichgraeber JF, Fleshman RG, Shaw JD, Chavarria C, Kau CH, Gateno J, Xia JJ. Long-term treatment outcome of presurgical nasoalveolar molding in patients with unilateral cleft lip and palate. J Craniofac Surg. 2011 Jan;22(1): 333-6. [Medline: 21239929] [doi: 10.1097/SCS.0b013e318200d874]

76. Peterson-Falzone SJ, Adali N, Mars M, Petrie A, Noar J, Somerlad B. Presurgical orthopedics has no effect on archform in unilateral cleft lip and palate. Cleft Palate Craniofac J. 2012;49(1):5-13. Cleft Palate Craniofac J. 2012 Nov;49(6):764-5. [Medline: 22591140] [doi: 10.1597/12-037]

77. Oliker, A. 3d Printing: Revolutionizing Medicine. Americas Quarterly. 2015;9(2), 46-7. [URL: http://www.americasquarterly.org]

78. Sutherland LM, Middleton PF, Anthony A, Hamdorf J, Cregan P, Scott D, Maddern GJ. Surgical simulation: a systematic review. Ann Surg. 2006 Mar;243(3):291-300. [Medline: 16495690] [PMC free article: 1448942] [doi: 10.1097/01.sla.0000200839.93965.26]

\section{To cite this article:}

Maillard S, Retrouvey JM, Ahmed MK, Taub PJ.

Correlation between Nasoalveolar Molding and Surgical, Aesthetic, Functional and Socioeconomic Outcomes Following Primary Repair Surgery: a Systematic Review

J Oral Maxillofac Res 2017;8(3):e2

URL: http:/www.ejomr.org/JOMR/archives/2017/3/e2/v8n3e2.pdf

doi: $10.5037 /$ jomr.2017.8302

Copyright (C) Maillard S, Retrouvey JM, Ahmed MK, Taub PJ. Published in the JOURNAL OF ORAL \& MAXILLOFACIAL RESEARCH (http://www.ejomr.org), 30 September 2017.

This is an open-access article, first published in the JOURNAL OF ORAL \& MAXILLOFACIAL RESEARCH, distributed under the terms of the Creative Commons Attribution-Noncommercial-No Derivative Works 3.0 Unported License, which permits unrestricted non-commercial use, distribution, and reproduction in any medium, provided the original work and is properly cited. The copyright, license information and link to the original publication on (http://www.ejomr.org) must be included. 